\title{
TENDENCIAS ACTUALES DE LAS MIGRACIONES EUROPEAS
}

\author{
ÓSCAR A. SANTACREU FERNÁNDEZ
}

Departamento de Sociología II. Universidad de Alicante (España).

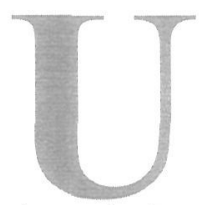

\section{RESUMEN}

na de las dimensiones que debe ser controlada cuando se estudian las migraciones internas de la Unión Europea es la de los factores de atracción y empuje (pull and push). A partir de una matriz de probabilidades de transición, el presente artículo aplica un modelo exploratorio de simulación del movimiento migratorio a partir de las actuales tendencias que permite contemplar, de una manera visual, las dinámicas internas de las migraciones europeas actuales, en las cuales los factores de atracción han ido ganado importancia frente a los factores de empuje.

Palabras clave: migraciones europeas, factores de atracción y empuje.

\section{INTRODUCCIÓN}

A pesar de los problemas que a lo largo de la historia han sacudido el continente europeo, la historia reciente de los países que lo conforman es una historia de encuentros. Así, junto a terribles guerras, el siglo pasado vio surgir una tendencia integracionista que dio lugar al Consejo de Europa y a la OTAN primero, y más tarde la Comunidad Económica Europea (CEE), a partir de la cual se incrementaron las iniciativas tendentes a la creación de una entidad única, en términos económicos y políticos, que han llevado a la Unión Europea que hoy conocemos y que actualmente está experimentando un importante proceso de ampliación. En este proceso de integración de la Unión Europea ha tenido una importancia decisiva el factor de las migraciones, las cuales, evidentemente, han ido evolucionando a lo largo de este proceso. Así, en los años 50 y 60 las migraciones adquirieron unas características distintas a las oleadas migratorias desde Europa hacia otros continentes tras la Segunda Guerra Mundial, y los países tradicionalmente emisores (Alemania, Francia, Suiza, Bélgica y Suecia) pasaron a ser receptores. Los países 
emisores pasaron a ser Italia, Grecia, España y Portugal, entonces poco industrializados, que proporcionaron grandes contingentes de mano de obra a los países industriales. Esta corriente migratoria sur-norte se vio interrumpida por la crisis económica de 1970, tras la cual las migraciones intraeuropeas cambiaron tanto cuantitativa como cualitativamente: coincidiendo con un aumento del nivel de renta y una disminución de la natalidad en la Europa mediterránea, estos países pasarán de ser emisores a receptores.

El presente artículo presenta una visión general de los movimientos migratorios en Europa durante el siglo XX y aplica un modelo exploratorio de simulación del movimiento migratorio, a partir de las actuales tendencias, que permite contemplar, de una manera visual, las dinámicas internas de las migraciones europeas actuales ${ }^{1}$.

\section{METODOLOGÍA}

El modelo exploratorio de simulación del movimiento migratorio que planteo en este trabajo está limitado por la naturaleza agregada de los datos secundarios empleados. Además, conviene llamar la atención sobre el hecho de que el modelo que aquí presento no pretende ser, en ningún modo, un modelo predictivo de población. Se trata de un modelo cuyo fin es simular y revelar las dinámicas internas de las migraciones en Europa, utilizando cadenas de Markov.

En primer lugar he desarrollado una Matriz de Probabilidades de Transición, a partir de los datos de residentes europeos en otros países de Europa ${ }^{2}$. Las cadenas de Markov son un método muy interesante para modelar el cambio de estado que se produce entre dos pasos. Esto es así especialmente cuando existe un conjunto de reglas subyacentes, como las que podemos encontrar en procesos como las migraciones.

La elaboración de modelos con cadenas de Markov implica ciertas limitaciones. En primer lugar, se entiende que la población está cerrada, en otras palabras, no entran ni salen individuos entre dos pasos del proceso que estudiamos. Del mismo modo, los coeficientes de transición son característicos de toda población y además son constantes a lo largo de todo el proceso. Como última restricción, se presupone que el

Este trabajo se apoya en el proyecto Competencia intercultural e integración de los residentes extranjeros europeos en la Comunidad Valenciana. Un análisis diagnóstico y prospectivo de los estereotipos económicos, sociales y politicos y su efecto sobre la inmigración como "pull factor", financiado por la Generalitat Valenciana, Conselleria de Cultura, Educación y Deporte, con referencia: CV04B-621. Investigador principal: prof. Antonio Alaminos.

2 Obviamente, el mejor método para modelar es un proceso de Markov aplicado a los datos anuales de migraciones. 
comportamiento de la movilidad sólo depende del estado actual y del previo, pero no de su historia, es decir, esta técnica utiliza memoria corta. A continuación defino los elementos que utilizaré en el análisis.

Los datos actuales de la población de los países estudiados se dispondrán en forma de Vector Distribución, de tal modo que se entenderá por población total la suma de las poblaciones de dichos países, y el Vector Distribución contemplará, para cada país, la proporción de su población respecto a la población total, de modo que la suma de los elementos del Vector Distribución sea igual a uno.

Se construirá una Matriz de Probabilidades de Transición que recoja las probabilidades de un individuo de pertenecer a la población extranjera de un determinado país, en un próximo paso del proceso, en función de su país de origen. Cumpliendo los requisitos de una Matriz de Probabilidades de Transición, todos los componentes de esta matriz son no negativos, y la suma de los componentes de cada fila es igual a uno.

Dado que trabajaré con cadenas de Markov, se operará mediante álgebra matricial, de modo que la situación del Vector Distribución en un paso posterior del proceso se obtendrá premultiplicando dicho Vector Distribución por la Matriz de Probabilidades de Transición construida. El Vector Distribución en un subsiguiente paso $n$ se obtendrá premultiplicando dicho Vector Distribución por la Matriz de Probabilidades de Transición elevada a $n$.

En cuanto a los datos utilizados, en el caso de la Matriz de Probabilidades de Transición partiré de los últimos datos disponibles sobre movilidad intraeuropea de los ciudadanos de la Unión Europea, extraídos de la New Cronos Database (Eurostat). Para la construcción del Vector Distribución utilizo los últimos datos disponibles de la población de extranjeros europeos en la Unión Europea, así como de la población total de cada uno de los países implicados, siendo la fuente de estos datos la New Cronos Database de Eurostat y las divisiones de Población y Estadísticas del Secretariado de Naciones Unidas.

\section{LOS FLUJOS INTERNOS MIGRATORIOS EUROPEOS}

Hoy en día, acostumbrados como estamos a aguantar estoicamente las colas de los controles de seguridad en los aeropuertos (ay de ti si has olvidado tu documentación), puede resultar chocante recordar que hasta finales del siglo XIX, en general, era posible desplazarse libremente por Europa. Claro, que entonces las migraciones no eran muy numerosas debido a la ausencia de las condiciones económicas necesarias. 
Las barreras a la inmigración fueron apareciendo junto a los controles de inmigración, los pasaportes y el surgimiento de derechos civiles, políticos y sociales específicos de cada nacionalidad. Durante el período de la posguerra se produjo un significativo número de migraciones desde los regímenes comunistas del este hacia el oeste. Así, antes de la construcción del Muro de Berlín en 1961, numerosos ciudadanos de Alemania del Este, polacos, checos y húngaros, se desplazaron hacia el oeste, si bien la principal dirección migratoria hacia y desde la Europa occidental durante los años 50 y 60 fue de sur a norte. Estas migraciones de sur a norte fueron frecuentemente iniciadas por trabajadores bajo la supervisión de los Estados a través de ministerios y consulados. Posteriormente, estos trabajadores fueron creando comunidades a las que se fueron sumando familiares, amigos y vecinos, cuyo desplazamiento no estaba necesariamente revisado por las instituciones estatales (E. Recchi, 2003: 5).

El crecimiento económico y la creciente industrialización de los años 60 en la Europa central y occidental llevó consigo la carencia de mano de obra para determinados trabajos de baja remuneración económica. A raíz de esta necesidad, numerosos inmigrantes se desplazaron desde los países del sur de Europa (Italia, España, Portugal y Grecia), más tarde desde Turquía y Yugoslavia y finalmente desde el Magreb y el Tercer Mundo hacia el norte y el este europeos para encontrar trabajo principalmente en sectores como la construcción, la industria y los servicios (Rodríguez Pose, 2002).

La ilustración 1 muestra los principales flujos de migración internacional laboral hacia los países de Europa occidental de 1945 a 1973.

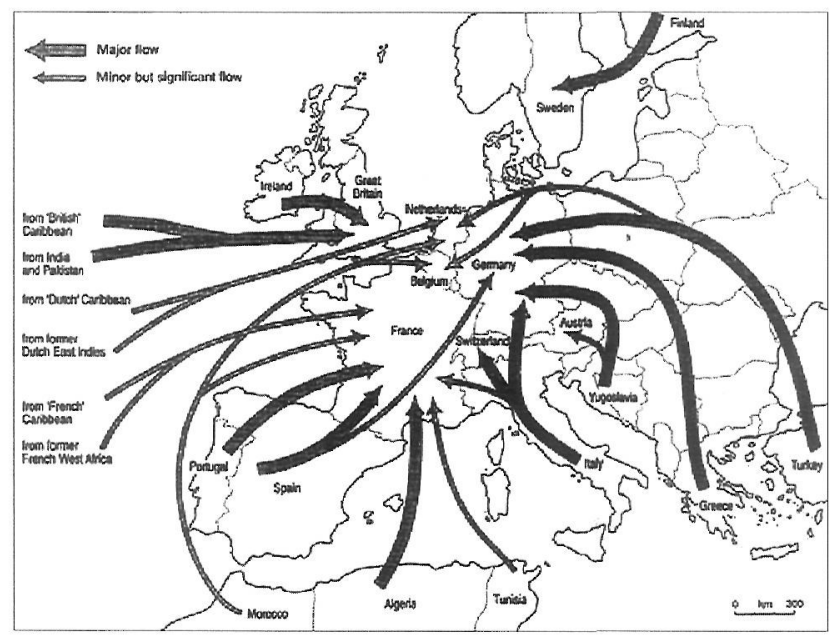

Fuente: Pinder (1998: 265) 
A finales de los años 60 , y como resultado de la posibilidad que se abrió a la migración de carácter familiar, la composición de los flujos migratorios comenzó a cambiar y pasó de una composición básica de trabajadores solteros a otra en la que encontramos más mujeres y niños implicados. La consecuencia lógica iba a ser que se pasaría de un modelo de migración temporal a un modelo de larga estancia o de permanencia, al instalarse núcleos familiares en los países de llegada. Estas migraciones estuvieron principalmente vinculadas a las cuotas esponsorizadas por los gobiernos y a los esquemas Gastarbeiter (E. Recchi, 2003:7).

Los antiguos flujos migratorios de sur a norte, de la Europa mediterránea a la Europa industrial, vinculados a migraciones laborales, van a ser reemplazados en los años 70 por una migración "de retorno". E. Recchi (2003) contempla tres nuevas formas principales de migración como resultado de las nuevas políticas más abiertas de control de la inmigración entre los estados miembros de la Comunidad Europea: en primer lugar un incremento de las migraciones internacionales de trabajadores cualificados, dentro de la Unión Europea y entre la Unión Europea y otras áreas de la economía global; en segundo lugar, un ligero aumento del número de emigrantes en busca de asilo, especialmente desde mediados de los 80; en tercer lugar, un aumento y diversificación de la migración secreta y/o ilegal. King $(1998,281)$ atribuye este aumento al cierre de los canales normales de entrada a Europa, y al aumento de los factores de empuje a la migración en otras regiones del mundo.

Durante los años 90 se ha configurado un nuevo mapa de las migraciones europeas, respondiendo a nuevos patrones y formas de movimiento. En esta década, la cantidad de extranjeros de la Unión Europea ha crecido sensiblemente (con la excepción de Grecia, Francia, Suecia e Italia), especialmente en Austria y Portugal, así como en Finlandia, España y Dinamarca. En otros países, como Reino Unido y Países Bajos, el incremento ha sido menor. En términos generales, la tendencia es bastante regular. Durante los años 90 la entrada de ciudadanos europeos en cada uno de estos países tiende a aumentar o a mantenerse estable (E. Recchi, 2003). Los últimos tres años de la década hubo ciertos cambios en Francia y Alemania, cuyos flujos de entrada disminuyeron sustancialmente, mientras que se observó una aceleración de estos flujos en Portugal y Suecia. Luxemburgo es el país donde el flujo de inmigrantes tiene una mayor proporción de emigrantes intra-europeos. En contraste, los inmigrantes europeos en Italia constituyen una pequeña fracción del total de extranjeros legalmente asentados en estos años. Parece que las migraciones de retiro, aquéllas que se producen al final de la vida 
laboral, prefieren otros países mediterráneos económicamente más accesibles, como España, Portugal y Grecia.

En resumen, estos autores plantean tres patrones principales de migración intraeuropea:

- El legado de los movimientos tradicionales sur-norte. Los datos permiten observar que la mayor parte de los extranjeros europeos residentes en países tradicionalmente importadores de mano de obra tienen como país de origen aquél que en su tiempo proporcionó esa mano de obra.

- Movimientos ligados a la proximidad geográfica y cultural, como, por ejemplo, los ciudadanos británicos en Irlanda o austríacos en Alemania.

- Migraciones por retiro profesional. Una sustancial parte de los ciudadanos de países del norte y del centro de Europa que residen en países del sur se han trasladado con una edad relativamente alta, en busca del buen tiempo o de la oferta de ocio. Tenemos un excepcional ejemplo en muchos pueblos de la provincia de Alicante, habitados en una gran medida por este tipo de ciudadanos europeos (alemanes, ingleses...).

Paralelamente, los años 90 han sido testigos del auge de dos nuevas tendencias en migración intraeuropea. Por un lado, la compuesta por trabajadores altamente cualificados. Por otro lado, los ya mencionados emigrantes que buscan las mejores condiciones para el retiro después de su vida laboral. En este sentido, Rodríguez Pose (2002: 98-100) describe el gran aumento de la migración de individuos altamente cualificados durante los años 90 . Identifica este autor dos factores principales en la migración de este tipo de trabajadores: la alta preparación de las nuevas generaciones europeas y la simplificación de los trámites burocráticos (2002: 99). En cuanto a la migración de personas jubiladas, parece ser que las personas que han estudiado, trabajado o vivido en el extranjero, o que han contraído matrimonio como inmigrantes, o que mantienen lazos familiares fuera de su país son más proclives a elegir su retiro profesional en el extranjero (E. Recchi, 2003). Según estos autores también el hecho de haber estado de vacaciones en el extranjero facilita el desarrollo de conocimiento y arraigos fuera de su país de origen. También es importante en este sentido la historia geopolítica, como en el caso del colonialismo británico.

Otro factor a tener en cuenta es el de los programas de formación superior en el extranjero, a modo de intercambio, como, por ejemplo, el Programa Erasmus. Este tipo de programas se ha visto favorecido, 
principalmente, por la necesidad planteada por la Comisión Europea del dominio de dos o más lenguas del espacio común europeo como requisito para la efectiva integración. Durante el período 1999-2000, los países con mayores tasas de intercambio fueron Reino Unido, Francia, España, Alemania e Italia (King y Ruiz Gelices 2003).

En resumen, las migraciones intraeuropeas pueden englobarse en distintos períodos históricos. Tras la segunda guerra mundial hasta los años setenta encontramos una migración relacionada con el movimiento de mano de obra desde el sur hacia el norte. En un período más reciente nos encontramos con otro tipo de migración más ligada al retiro laboral, los estudios y el intercambio de trabajadores altamente cualificados. En cuanto a los motivos para la emigración, la desigualdad económica pasa el relevo al factor de la libre circulación de personas como derecho.

En este relativamente nuevo escenario de libre circulación no está de más tener en cuenta los procesos de identidad como importantes factores en un análisis abierto de los movimientos migratorios. Es importante definir estos procesos de identidad en una Europa con migraciones y movilidad espacial de sus ciudadanos (trabajadores, jubilados, estudiantes, turistas...), atendiendo a los distintos tipos de procesos de identidad étnica, cultural o social (O. Santacreu y M.C. Albert, 2004). De hecho, el actual proceso de ampliación de la Unión Europea pone de manifiesto la importancia de elementos como la identidad o la idea de ciudadanía, entendida como "un status concedido a todos aquellos que son miembros plenos de la comunidad. Todos aquellos que tienen el status son iguales respecto a los derechos y deberes que acompañan al status." (T. H. Marshall, 1997). Desde un punto de vista institucional, "la ampliación de la Unión Europea supone un logro histórico que pondrá fin a la división artificial del continente y traerá consigo una Europa más fuerte y estable, capaz de aprovechar plenamente las posibilidades de un mercado interior de 500 millones de personas ${ }^{3 " .}$. La consolidación de los valores europeos en sus ciudadanos está en la base de la propuesta unificadora, frente a la premisa original basada en la libertad de movimientos de capital, servicios, ideas y personas, aunque desde una Europa dividida (Comisión Europea, 2001). Estos valores, así como la existencia de intereses comunes, son los que van a conformar los puntos de referencia de la identidad, por encima de otras consideraciones como la proximidad o la nacionalidad, determinando la emergencia de "comunidades virtuales" (A. Alaminos, 2002: 332). 


\section{LA IDENTIDAD EN LOS PAÍSES EUROPEOS}

En un trabajo anterior (O. Santacreu y M.C. Albert, 2004) planteo que el análisis de la identidad sugiere una estructura dimensional de primer orden conformada por dimensión simbólica, dimensión espacial y dimensión pragmática. La primera engloba variables relacionadas con la valoración de la imagen europea y de la pertenencia a la misma. La dimensión espacial se refiere a la vertebración del sentido de pertenencia a la propia región, ciudad y nación. La dimensión pragmática, por su parte, abarca aquellos aspectos que hacen referencia a la valoración, en términos de coste y beneficio, de la pertenencia a la Unión Europea, siendo un factor en cierta medida opuesto a la dimensión simbólica. Encontramos también una estructura dimensional de segundo orden que se corresponde con la doble articulación planteada por Alaminos, acorde con la distinción entre identidad metafórica e identidad metonímica (A. Alaminos, 2002). En este sentido, la identidad metafórica hace referencia a la idea de "estar incluido en" un todo de banderas, ideas y símbolos. La identidad metonímica europea se refiere, por contra, a la idea de formar parte de algo, "ser parte de", y se articula entre el ámbito local-nacional y el ámbito extranjero (Unión Europea), expresando el grado de distancia entre los ámbitos espaciales considerados (local, regional, nacional, europeo). Tomando estas dos dimensiones, es posible disponer los países europeos en un mapa bidimensional, tal y como se presenta en la ilustración 2.

Ilustración 2. Identidad metafórica e identidad metonímica

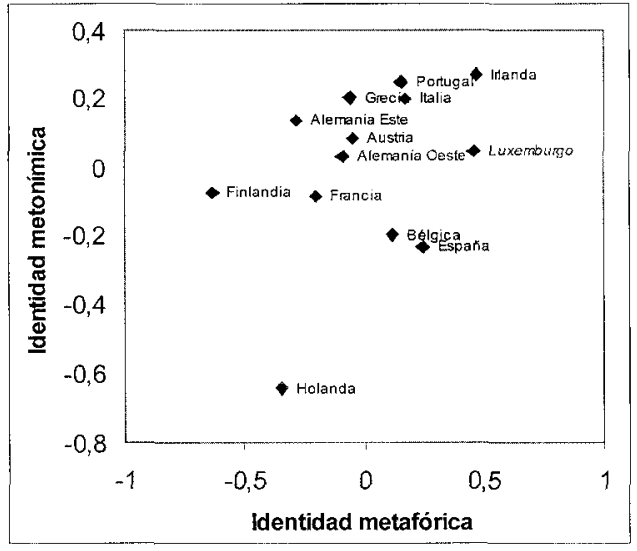

Fuente: O. Santacreu y M.C. Albert, 2004

Tal y como se observa en la ilustración, si atendemos a la identidad entendida como la idea de "estar incluidos" (identidad metafórica), encontramos que Bélgica, Portugal, Italia, España, Irlanda y Luxemburgo 
puntúan por encima de la media. Para estos países, estar en Europa significa "estar incluidos". Por debajo de la media puntúan, como vemos, países del centro y del norte de Europa como Austria, Alemania, Francia, Holanda y Finlandia. En cuanto a identidad metonímica, articulada en lo próximo (ámbito local y nacional) y lo extranjero (la UE), se puede observar una identidad metonímica más alta en aquellos países con verticalidad menor en la vertebración horizontal de los ámbitos espaciales considerados (por ejemplo, Italia), mientras que una identidad metonímica baja implica una mayor verticalidad o distancia entre los distintos ámbitos espaciales (por ejemplo, España).

Más interesante resulta comparar el posicionamiento de estos países en términos de dimensión simbólica y dimensión pragmática. En particular, si dividimos el espacio del mapa en cuatro partes, tomando como ejes divisores las puntuaciones medias en ambas dimensiones, podremos entender mejor el significado transmitido por el diagrama. La ilustración 3 recoge la posición de cada uno de los países analizados en este mapa bidimensional, mientras que la ilustración 4 divide el espacio en cuatro partes diferenciadas: países con alto componente simbólico y bajo componente pragmático, países con alto componente pragmático y simbólico, países con bajo componente pragmático y simbólico y países con alto componente pragmático y bajo componente simbólico.

Ilustración 3. Dimensión simbólica y dimensión pragmática

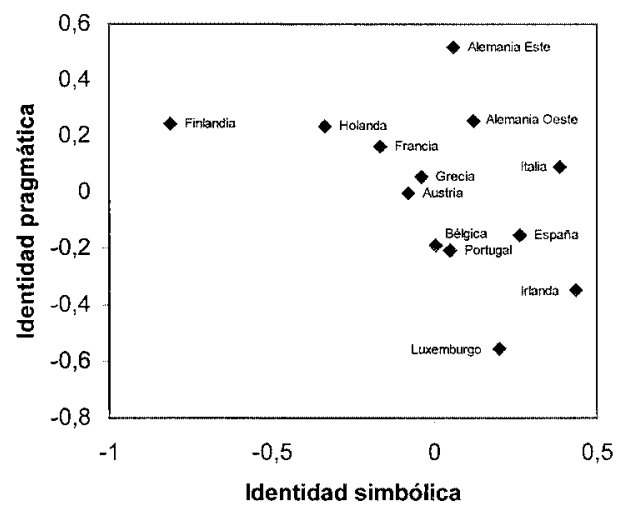

Fuente: O. Santacreu y M.C. Albert, 2004

Como en el caso anterior, Irlanda, España, Luxemburgo, Bélgica y Portugal obtienen una alta puntuación en la dimensión simbólica. Para algunos países, como Alemania y, en menor grado, Grecia, Austria o Francia, el componente simbólico también es importante, aunque predomina en ellos una dimensión pragmática, especialmente importante 
en el caso de Holanda y Finlandia, cuya adscripción a la Unión Europea obedece básicamente a una motivación racional. Como era de esperar, los países que forman parte del núcleo fundador del mercado único del carbón y el acero que está en el origen de la Comunidad Económica Europea presentan una idea de Europa en la que predomina el componente pragmático (económico) frente al componente simbólico. Los países que se incorporaron más tarde, Luxemburgo y los países mediterráneos, buscan no sólo dicha dimensión pragmática, sino también el prestigio de formar parte del club y de sus connotaciones de estabilidad y regeneración democrática.

Ilustración 4. División del espacio simbólico/pragmático

\begin{tabular}{|c|c|c|}
\hline $\begin{array}{l}+ \\
\mathbf{P} \\
\mathbf{R} \\
\mathbf{A} \\
\mathbf{G} \\
\mathbf{M}\end{array}$ & $\begin{array}{l}\text { Alto componente pragmático } \\
\text { Bajo componente simbólico }\end{array}$ & Alto componente pragmático y simbólico \\
\hline $\begin{array}{l}\text { T } \\
\text { I } \\
\text { C } \\
\text { A }\end{array}$ & Bajo componente pragmático y simbólico & $\begin{array}{l}\text { Alto componente simbólico } \\
\text { Bajo componente pragmático }\end{array}$ \\
\hline & S I M B & L I CA \\
\hline
\end{tabular}

Fuente: O. Santacreu y M.C. Albert, 2004

Así pues, en términos de identidad vamos a encontrar en Europa tres grupos principales de países: en primer lugar, los países como Reino Unido, Suecia, Dinamarca y Finlandia donde hay un mayor recelo hacia la Unión Europea, desconfianza más o menos manifiesta en sus instituciones, su disponibilidad al uso de la moneda común, o su mayor sentimiento de adhesión a la propia nacionalidad. En segundo lugar, los países del núcleo fundador de la Unión Europea (Alemania y Francia) con una identidad europea más basada en el componente pragmático que en el componente simbólico (bandera, moneda, instituciones, ciudadanía o ámbitos espaciales). Por último, podemos agrupar a los países de incorporación más tardía a la Unión Europea y cuyas motivaciones difieren de las del núcleo fundador, estando más orientadas hacia un componente simbólico de la identidad europea (países como España, Italia y Luxemburgo).

En esta primera parte he intentado ofrecer una visión general de las migraciones en Europa, así como de las dimensiones que conforman la identidad europea. A continuación, aplicaré un modelo exploratorio de simulación del movimiento migratorio a partir de las actuales tendencias que, siempre que se consideren las naturales limitaciones del modelo, pueden ayudar a comprender de una manera visual las actuales dinámicas internas de las migraciones europeas. 


\section{MODELO DE SIMULACIÓN DE MOVILIDAD INTERNA}

Como se ha indicado anteriormente, para la construcción de la Matriz de Probabilidades de Transición necesaria para trabajar con cadenas de Markov parto de los datos de la movilidad intraeuropea de los ciudadanos de la Unión Europea. La fuente de los datos es la New Cronos Database (Eurostat).

Tabla 1. Movilidad intra-europea de los ciudadanos de la UE, último año disponible. Flujos migratorios por nacionalidad en porcentaje sobre el flujo total de ciudadanos europeos.

\begin{tabular}{|c|c|c|c|c|c|c|c|c|c|c|c|c|c|c|}
\hline \multirow{3}{*}{$\begin{array}{l}\text { NACIONALIDAD } \\
\text { DE LOS } \\
\text { EXTRANJEROS } \\
\text { EUROPEOS } \\
\end{array}$} & \multicolumn{14}{|c|}{ PAÍS DE DESTINO } \\
\hline & A & B & DK & FIN & $\mathbf{F}$ & D & I & L & NL & P & E & $\mathrm{S}$ & UK & Tot \\
\hline & 1998 & 1999 & 1998 & 1999 & 1998 & 1999 & 1999 & 1999 & 1998 & 1998 & 1998 & 1998 & 1998 & \\
\hline Austria & - & 0,90 & 2,10 & 1,80 & 1,00 & 8,80 & 4,60 & 0,50 & 1,80 & 1,20 & 1,50 & 1,10 & 0,10 & 4,20 \\
\hline Bélgica & 1,40 & & 1,90 & 1,10 & 6,70 & 1,50 & 3,50 & 16,40 & 9,70 & 3,70 & 5,80 & 1,10 & 1,20 & 2,60 \\
\hline Dinamarca & 1,70 & 1,40 & - & 4,50 & 1,40 & 1,80 & 2,10 & 2,00 & 2,00 & 0,90 & 1,40 & 13,40 & 3,80 & 2,40 \\
\hline Finlandia & 2,60 & 1,50 & 5,00 & - & 1,10 & 2,10 & 2,00 & 1,00 & 2,50 & 1,00 & 3,30 & 35,90 & 2,40 & 3,10 \\
\hline Francia & 5,10 & 28,30 & 9,60 & 7,00 & & 11,30 & 19,60 & 26,60 & 10,30 & 15,70 & 12,10 & 7,20 & 22,00 & 15,00 \\
\hline Alemania & 52,70 & 11,00 & 20,90 & 12,40 & 10,70 & - & 24,20 & 8,50 & 23,80 & 22,00 & 31,90 & 13,70 & 13,30 & 11,50 \\
\hline Italia & 10,40 & 9,30 & 6,80 & 4,90 & 13,80 & 25,80 & 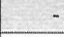 & 6,70 & 6,90 & 7,60 & 8,90 & 3,50 & 14,20 & 16,40 \\
\hline Luxemburgo & 0,30 & 0,70 & 0,00 & 0,00 & 0,30 & 0,50 & 0,10 & - & 0,10 & 0,30 & 0,10 & 0,00 & 0,00 & 0,30 \\
\hline Países bajos & 4,20 & 22,10 & 7,60 & 3,80 & 3,10 & 4,80 & 4,50 & 2,70 & - & 6,90 & 4,90 & 4,20 & 4,20 & 5,80 \\
\hline Portugal & 3,20 & 4,70 & 1,20 & 0,30 & 31,9 & 10,90 & 3,60 & 25,10 & 3,70 & - & 6,40 & 0,80 & 3,60 & 7,70 \\
\hline España & 2,40 & 4,20 & 6,40 & 3,10 & 9,20 & 6,10 & 10,60 & 1,30 & 5,80 & 18,70 & - & 3,40 & 9,80 & 6,20 \\
\hline Suecia & 3,40 & 2,00 & 18,40 & 44,60 & 2,50 & 2,50 & 3,00 & 1,70 & 3,30 & 2,30 & 2,40 & - & 4,40 & 3,50 \\
\hline Reino Unido & 7,60 & 10,80 & 16,80 & 12,90 & 15,10 & 8,90 & 13,30 & 5,20 & 23,80 & 18,70 & 20,40 & 11,80 & - & 9,50 \\
\hline Total & 100,00 & 100,00 & 100,00 & 100,00 & 100,00 & 100,00 & 100,00 & 100,00 & 100,00 & 100,00 & 100,00 & 100,00 & 100,00 & 100,00 \\
\hline
\end{tabular}

Fuente: Eurostat New Cronos Database

Las casillas sombreadas muestran los principales países de origen para cada país de destino. En este sentido, los principales flujos migratorios internos actuales en Europa, en términos de qué países son los principales aportadores de migración interna, son los siguientes:

- Austria recibe entradas principalmente desde Alemania.

- Bélgica desde Francia y Países Bajos.

- Dinamarca desde Alemania, Suecia y Reino Unido.

- Finlandia recibe principalmente desde Suecia.

- Francia desde Portugal.

- Alemania desde Italia.

- Italia recibe principalmente de Alemania, España y Reino Unido.

- Luxemburgo desde Francia, Bélgica y Portugal.

- Países Bajos desde Reino Unido y Alemania.

- Portugal desde Alemania, Reino Unido y España. 
- España desde Alemania, Reino Unido y Francia.

- Suecia desde Finlandia.

- Reino Unido desde Francia, Italia y Alemania.

Se perciben aquí las direcciones de los flujos migratorios antes comentadas. Por otro lado, para la construcción del Vector Distribución utilizo los datos de la población de extranjeros europeos en la Unión Europea, así como de la población total de cada uno de los países implicados:

Tabla 2. Extranjeros en la UE y población

\begin{tabular}{|l|c|c|c|c|c|c|c|c|c|c|c|c|c|}
\hline Países & A & B & DK & FIN & F & D & I & L & NL & P & E & S & UK \\
\hline $\begin{array}{l}\text { Extranjeros europeos } \\
\text { (\% de total extranjeros) }\end{array}$ & 13,0 & 62,2 & 20,5 & 18,7 & 36,6 & 25,1 & 13,7 & 89,0 & 28,0 & 26,3 & 42,7 & 33,9 & 18,5 \\
\hline $\begin{array}{l}\text { Extranjeros europeos } \\
\text { (\% de total población) }\end{array}$ & 1,2 & 5,5 & 1 & 0,3 & 2 & 2,3 & 0,3 & 31 & 1,2 & 0,5 & 0,7 & 2 & 0,7 \\
\hline Población estimada & 19730 & 10319 & 5364 & 5207 & 60144 & 82476 & 57423 & 453 & 16149 & 10062 & 41061 & 8877 & 59251 \\
\hline Total extranjeros UE & 236,76 & 567,545 & 53,64 & 15,621 & 1202,88 & 1896,948 & 172,269 & 140,43 & 193,788 & 50,31 & 287,427 & 177,54 & 414,757 \\
\hline
\end{tabular}

Fuentes: Eurostat New Cronos Database y División de Población y Estadísticas del Secretariado de Naciones Unidas. Nota: Los extranjeros de la UE se refieren a 1998. La población estimada se refiere a 2003.

Estos datos tienen una doble utilidad. Además de permitir la elaboración del Vector Distribución (tabla 3), permiten pasar la anterior tabla de movilidad (tabla 1) de porcentajes columna a porcentajes fila, a partir de los cuales podré por fin obtener la Matriz de Probabilidades de Transición.

Tabla 3. Vector distribución (para Cadenas de Markov)

\begin{tabular}{|l|c|c|c|c|c|c|c|}
\hline País & A & B & DK & FIN & F & D & I \\
\hline Población & 0,052401 & 0,027407 & 0,014246 & 0,013829 & 0,159738 & 0,219050 & 0,152511 \\
\hline Pob.+Extr. & 0,052314 & 0,028494 & 0,014198 & 0,013690 & 0,160727 & 0,220448 & 0,150953 \\
\hline País & L & NL & P & E & S & UK & TOTAL \\
\hline Población & 0,001203 & 0,042891 & 0,026724 & 0,109055 & 0,023577 & 0,157366 & 1,000000 \\
\hline Pob.+Extr. & 0,001547 & 0,042813 & 0,026509 & 0,108393 & 0,023719 & 0,156193 & 1,000000 \\
\hline
\end{tabular}

Fuente: elaboración propia a partir de Eurostat New Cronos Database y División de Población y Estadísticas del Secretariado de Naciones Unidas.

Para pasar los datos de la tabla de movilidad (tabla 1) a valores absolutos, utilizaré los datos de población total de los países de la Unión Europea que son incluidos en este estudio.

Una vez disponemos de los datos en términos absolutos, es fácil construir una Matriz de Probabilidades de Transición expresando en término de probabilidades dichos datos. La tabla 5 presenta la Matriz de Probabilidades de Transición así construida. 
Tabla 4. Datos absolutos basados en tablas 1 y 2.

\begin{tabular}{|c|c|c|c|c|c|c|c|c|c|c|c|c|c|c|}
\hline \multirow{3}{*}{$\begin{array}{l}\text { NACIONALIDAD } \\
\text { DE EXTRANJEROS } \\
\text { EUROPEOS }\end{array}$} & \multicolumn{14}{|c|}{ PAÍS DE DESTINO } \\
\hline & $\mathrm{A}$ & B & DK & FIN & $\mathbf{F}$ & D & I & $\mathrm{L}$ & NL & $\mathbf{P}$ & $\mathbf{E}$ & $\mathbf{S}$ & & \\
\hline & 1998 & 1999 & 1998 & 1999 & 1998 & 1999 & 1999 & 1999 & & 1998 & 1998 & & 1998 & \\
\hline & 19730 & 5,11 & 1,13 & 0,28 & 12,03 & & 7,92 & 0,70 & 3,49 & 0,60 & 4,31 & 1,95 & 0,41 & \\
\hline Bélg & 3,31 & 0319 & 1,02 & 0,17 & 80,59 & 28,45 & 6,03 & 23,03 & 18,80 & 1,86 & 16,67 & 1,95 & 4,98 & \\
\hline & & 7,95 & & & & 34,15 & 3,62 & 2,81 & 3,88 & 0,45 & & 23,79 & 15,76 & \\
\hline & &, 51 & & 5207 & 13,23 & 39,84 & 3,45 & 1,40 & 4,84 & 0,50 & & 63,74 & 9,95 & \\
\hline & 2,07 & 0,62 & & 1,09 & 0144 & 214,36 & 33,76 & 37,35 & 19,96 & 7,90 & & 12,78 & 91,25 & \\
\hline & 4,77 & 2,43 & 11,21 & 1,94 & 128,71 & 82476 & 41,69 & 11,94 & 46,12 & 11,07 & & 24,32 & 55,16 & \\
\hline Italia & 24,62 & 52,78 & 3,65 & 0,77 & 166,00 & 489,41 & 57423 & 9,41 & 13,37 & 3,82 & 25,58 & 6,21 & 58,90 & \\
\hline Luxemb & 0,71 & 3,97 & 0,00 & 0,00 & 3,61 & 9,48 & 0,17 & 453 & 0,19 & 0,15 & 0,29 & 0,00 & 0,00 & 471,58 \\
\hline Países bajos & 9,94 & 125,43 & 4,08 & 0,59 & 37,29 & 91,05 & 7,75 & 3,79 & 16149 & 3,47 & 14,08 & 7,46 & 17,42 & 1617136 \\
\hline & & 26,67 & & 0,05 & 383,72 & 206,77 & 6,20 & 35,25 & 7,17 & 10062 & 18,40 & 1,42 & 14,93 & \\
\hline España & 5,68 & 23,84 & 3,43 & 0,48 & 110,66 & 115,71 & 18,26 & 1,83 & 11,24 & 9,41 & 41061 & 6,04 & 40,65 & 41408,23 \\
\hline Suecia & 8,05 & 11,35 & & 6,97 & 30,07 & 47,42 & 5,17 & 2,39 & 6,40 & 1,16 & 6,90 & 8877 & 18,25 & 9030,99 \\
\hline Reino Unido & 17,99 & 61,29 & 9,01 & 2,02 & 181,63 & 168,83 & 22,91 & 7,30 & 46,12 & 9,41 & 58,64 & 20,95 & 59251 & 59857,11 \\
\hline TOTAL & 19954,92 & 10868,95 & 5415,87 & 5222,06 & 61308,39 & 84088,41 & 57579,94 & 590,20 & 16330,58 & 10111,81 & 41345,84 & 9047,62 & 59578,66 & 381443,23 \\
\hline
\end{tabular}

Fuente: elaboración propia a partir de Eurostat New Cronos Database y División de Población y Estadísticas del Secretariado de Naciones Unidas

Tabla 5. Matriz de Probabilidades de Transición (para Cadena de Markov)

\begin{tabular}{|c|c|c|c|c|c|c|c|c|c|c|c|c|c|c|}
\hline \multirow{3}{*}{$\begin{array}{l}\text { NACIONALIDAD } \\
\text { DE } \\
\text { EXTRANJEROS } \\
\text { EUROPEOS } \\
\end{array}$} & \multicolumn{14}{|c|}{ PAÍS DE DESTINO } \\
\hline & $\mathrm{A}$ & B & DK & FIN & $\mathbf{F}$ & D & I & $\mathrm{L}$ & $\mathrm{NL}$ & $\mathbf{P}$ & $\mathrm{E}$ & $\mathbf{S}$ & UK & \multirow{2}{*}{ TOT } \\
\hline & 1998 & 1999 & 1998 & 1999 & 1998 & 1999 & 1999 & 1999 & 1998 & 1998 & 1998 & 1998 & 1998 & \\
\hline Austria & 0,98972 & 0,00026 & 0,00006 & 0,00001 & 0,00060 & 0,00837 & 0,00040 & 0,00004 & 0,00017 & 0,00003 & 0,00022 & 0,00010 & 0,00002 & 1,00 \\
\hline Bélgica & 0,00032 & 0,98221 & 0,00010 & 0,00002 & 0.00767 & 0,00271 & 0,00057 & 0.00219 & 0.00179 & 0,00018 & 0.00159 & 0,00019 & 0,00047 & 1,00 \\
\hline Dinamarea & 0,00073 & 0,00145 & 0.97848 & 0,00013 & 0,00307 & 0.00623 & 0,00066 & 0,00051 & 0,00071 & 0,00008 & 0.00073 & 0,00434 & 0,00288 & 1,00 \\
\hline Finlandia & 0,00115 & 0.00159 & 0,00050 & 0,96950 & 0,00246 & 0,00742 & 0,00064 & 0,00026 & 0,00090 & 0,00009 & 0,00177 & 0,01187 & 0,00185 & 1,00 \\
\hline Francia & 0,00020 & 0,00264 & 0,00008 & 0,00002 & 0,98962 & 0,00353 & 0,00056 & 0,00061 & 0,00033 & 0,00013 & 0,00057 & 0,00021 & 0,00150 & 1,00 \\
\hline Alemania & 0,00150 & 0,00075 & 0,00013 & 0,00002 & 0,00155 & 0,99265 & 0,00050 & 0,00014 & 0,00056 & 0,00013 & 0,00110 & 0,00029 & 0,00066 & 1,00 \\
\hline Italia & 0,00042 & 0,00091 & 0,00006 & 0,00001 & 0,00285 & 0,00840 & 0,98534 & 0,00016 & 0,00023 & 0,00007 & 0,00044 & 0,00011 & 0,00101 & 1,00 \\
\hline Luxemburgo & 0,00151 & 0,00842 & 0,00000 & 0,00000 & 0,00765 & 0,02011 & 0,00037 & 0,96060 & 0,00041 & 0,00032 & 0,00061 & 0,00000 & 0,00000 & 1,00 \\
\hline Paises bajos & 0,00060 & 0,00761 & 0,00025 & 0,00004 & 0,00226 & 0,00553 & 0,00047 & 0,00023 & 0,98043 & 0,00021 & 0,00086 & 0,00045 & 0,00106 & 1,00 \\
\hline Portugal & 0,00070 & 0,00248 & 0,00006 & 0,00000 & 0,03563 & 0,01920 & 0,00058 & 0,00327 & 0,00067 & 0,93419 & 0,00171 & 0,00013 & 0,00139 & 1,00 \\
\hline España & 0,00014 & 0,00058 & 0,00008 & $0,0000 \mathrm{I}$ & 0,00267 & 0,00279 & 0,00044 & 0,00004 & 0,00027 & 0,00023 & $0,99 \mid 61$ & 0,00015 & 0,00098 & 1,00 \\
\hline Suecia & 0,00089 & 0,00126 & 0,00109 & 0,00077 & 0,00333 & 0,00525 & 0,00057 & 0,00026 & 0,00071 & 0,00013 & 0,00076 & 0,98295 & 0,00202 & 1,00 \\
\hline Reino Unido & 0,00030 & 0,00102 & 0,00015 & 0.00003 & 0,00303 & 0,00282 & 0,00038 & 0,00012 & 0,00077 & 0,00016 & 0,00098 & 0,00035 & 0,98987 & 1,00 \\
\hline
\end{tabular}

Fuente: elaboración propia a partir de Eurostat New Cronos Database y División de Población y Estadisticas del Secretariado de Naciones Unidas.

Partiendo del Vector Distribución de la tabla 3, si premultiplicamos sucesivamente por la Matriz de Probabilidad de Transición (tabla 5) elevada a $1,2,3 \ldots$, vamos a obtener una estimación del Vector Distribución a 1, 2, 3...n pasos. En lugar de presentar aquí la serie numérica del Vector Distribución, resulta más interesante graficar dichos resultados. La ilustración 4 muestra de forma gráfica la evolución del Vector Distribución a lo largo del modelo de simulación.

Se observa en la ilustración que países como Francia y Alemania, de mantenerse sin cambios la actual dinámica y eliminando otras posibles fuentes de variabilidad, seguirían estando a la cabeza de la población, 
Ilustración 4. Modelo de simulación de movilidad interna

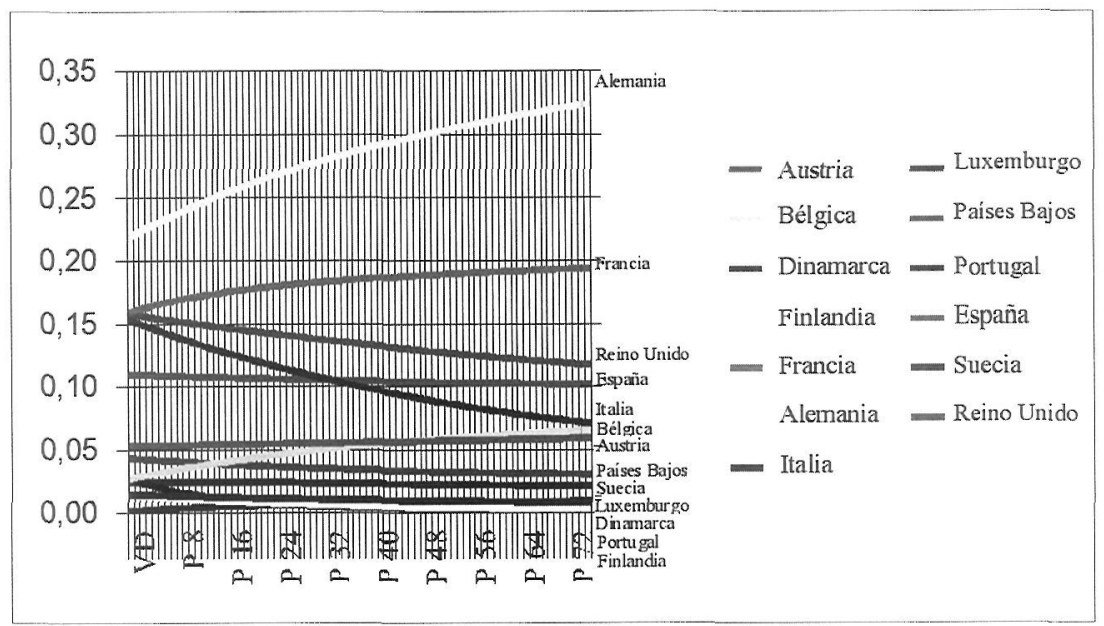

Fuente: elaboración propia

Nota: Modelo para revelar la dinámica interna de los procesos.

manteniendo un saldo migratorio favorable. Reino Unido e Italia, en cambio, partiendo de un nivel similar al de Francia, muestran un saldo migratorio desfavorable que hace que descienda su población en estas condiciones controladas. En menor medida presentan un saldo migratorio negativo Portugal y Países Bajos. Bélgica y España, por su parte, presentan un saldo migratorio favorable que les haría situarse, en términos de población, en una situación proporcionalmente superior a la actual. Por otro lado, si planteamos una situación de inicio equiprobable en la que todos los países parten en igualdad de población, nos encontraríamos con la situación que plantea la ilustración 5 .

Al partir de una situación equiprobable, la ilustración muestra con mayor claridad la dinámica interna del modelo, pudiendo fácilmente distinguir entre aquellos países con saldo migratorio favorable (Alemania, Francia, Bélgica, España, Reino Unido) y aquéllos que presentan una estabilización (como Austria) o un saldo negativo más o menos acusado (Suecia, Italia, Países Bajos, Dinamarca, Luxemburgo, Finlandia y Portugal). Llevando el modelo al extremo, esto es, más allá de la estabilización de la Matriz de Probabilidades de Transición ${ }^{4}$, obtenemos un gráfico (ilustración 6) que muestra más claramente esta tendencia.

4 Las sucesivas potencias de la Matriz de Probabilidades de Transición provocan su estabilización. Con la Matriz de Probabilidades de Transición estabilizada, la situación final del Vector Distribución es independiente de su situación inicial. 
Ilustración 5. Modello de simulación de inicio equiprobable

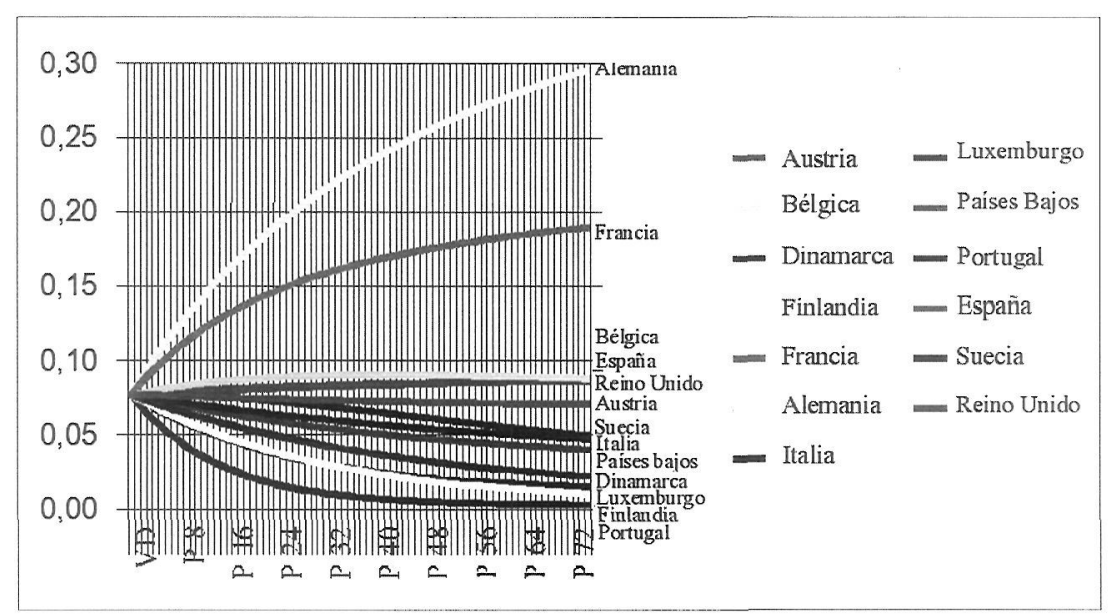

Fuente: elaboración propia

Nota: Modelo para revelar la dinámica interna de los procesos.

Ilustración 6. Modelo extremo de simulación de inicio equiprobable

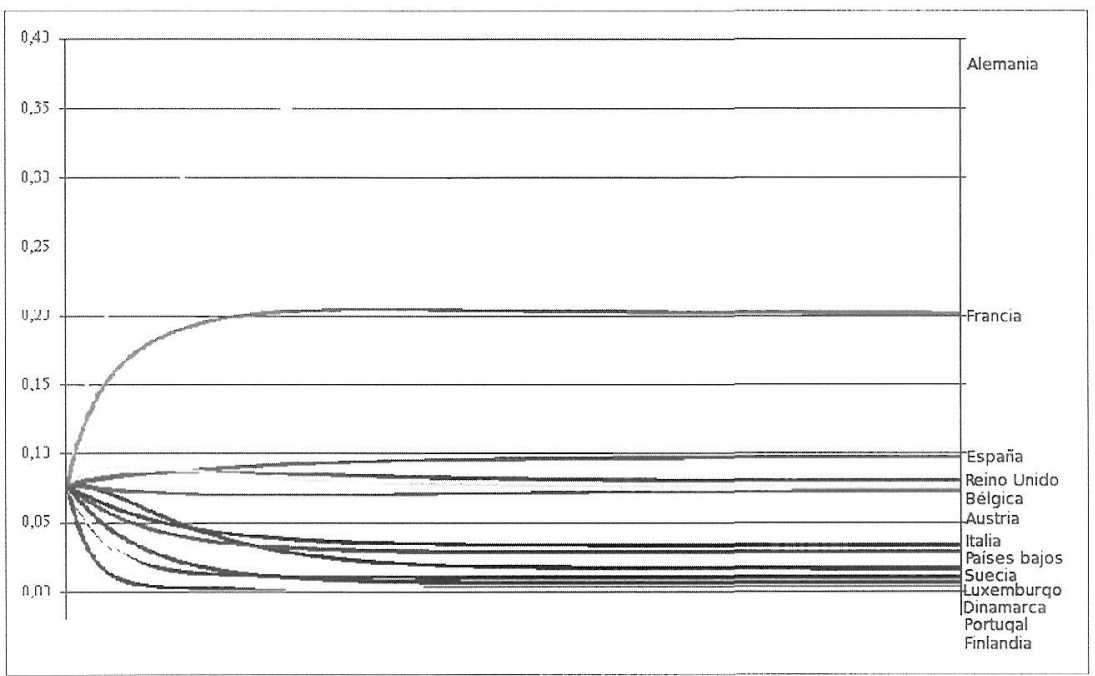

Fuente: elaboración propia

Nota: Modelo para revelar la dinámica interna de los procesos Conclusiones

El modelo de simulación que he planteado es un reflejo del período histórico actual en las migraciones europeas, en el cual aparecen, junto a los tradicionales flujos migratorios de sur a norte, las nuevas 
tendencias referidas a los nuevos perfiles de emigrantes: profesionales altamente cualificados y personas que buscan el merecido descanso tras finalizar su vida laboral. Estos nuevos perfiles difieren sensiblemente en sus motivaciones de, por ejemplo, aquellos trabajadores que se desplazan por necesidad en busca de un futuro mejor. En este sentido, existe una dimensión que por su importancia debe ser controlada cuando se estudian las migraciones internas de la Unión europea; me refiero a los factores de atracción y empuje (en inglés, pull and push). Tal y como se ha expuesto, históricamente las migraciones en Europa han tenido en su mayor parte un elemento forzado. Así, en la base de las migraciones de los años sesenta y setenta podemos encontrar exilios políticos o económicos, principalmente desde el sur de Europa. En este sentido, la actual composición de los ciudadanos europeos que viven en un país diferente de su país de origen contiene un componente importante de estas migraciones "empujadas" (push). En la actualidad, por contra, comienza a surgir un incremento evidente en el peso de los factores de atracción (pull).

En este sentido, es particularmente ilustrativo el caso de España, un país tradicionalmente emisor y que, según el modelo de simulación aquí planteado, pasa a ser un país receptor gracias sobre todo a las migraciones de retiro. Así, en la expresión gráfica del efecto de la Matriz de Probabilidades de Transición, podemos apreciar, especialmente en el modelo extremo (ilustración 6), las migraciones tradicionales a Francia o Alemania. Probablemente, esta dinámica interna permanecerá por la ampliación de la Unión Europea, especialmente en el caso alemán. Lo que resulta inusual, incluso en un estado preliminar, es la creciente población de ciudadanos extranjeros de la Unión Europea en países como España. En la simulación, la magnitud de este proceso es suficientemente fuerte para cancelar el efecto de la tradición española de emigración. Este nuevo proceso migratorio tiene una naturaleza diferente del anterior. Si tradicionalmente las migraciones forzosas fueron de sur a norte, estas nuevas formas de movilidad son principalmente de norte a sur.

En este sentido, podemos concluir que la subpoblación que define nuestro objetivo de investigación es probablemente representativa de una nueva mentalidad europea. En esta nueva cultura, parece que la movilidad no será más un indicador de drama social, sino una expresión de la libertad natural y en la que adquieren un peso importante las distintas dimensiones (simbólica, pragmática...) de la identidad europea. 


\section{BIBLIOGRAFÍA}

ALAMINOS, A. 2002. "Maneras de vivir la sociedad en Europa" en José Félix Tezanos (ed), Clase, estatus y poder en las sociedades emergentes, Madrid, Sistema. 2002

ALAMINOS, A. 2002. "Imágenes en Europa", en Gianfranco Bettin (Ed.), Mutamenti in Europa, Monduzzi, Bolonia.

Comisión Europea. 2001. La Unión Europea sigue creciendo. Bruselas, Colección Europa en movimiento.

BALDONI, E. 2003. The Free Movement of Persons in the EU: A Legal historical Overview, PIONEUR working paper 2003/2, Florence, CIUSPO.

Eurostat. 2002. "Migration Keeps the EU Population Growing", Statistics in Focus Eurostat, 7, 14.

KING, R. 1998. "From Guestworkers to Immigrants: Labour Migration from the Mediterranean to the Periphery", en Pinder, D. (ed.), The New Europe: Economy, Society and Environment, New York, Wiley and Sons.

KING, R. y RUIZ GELICES, E. 2003. "International Student Migration and the European Year Abroad : Effects on European Identity and Subsequent Migration Behaviour", International Journal of Population Geography, 9, 229-252.

KING, R., WARNES, T. y WILLIAMS, A. 1998. "International retirement migration in Europe", International Journal of Population Geography, 4, 91-111.

LIEBERSON, S. 1969. "Measuring Population Diversity", American Sociological Review, 34, 850-862.

MARSHALL, T.H. 1997. Ciudadania y clase social, REIS, 79:244-297.

PINDER, D. (ed.) 1998. The New Europe: Economy, Society and Environment, New York, Wiley and Sons.

POULAIN, M. 1996. "Migration Flows Between the Countries of the European Union: Current Trends", en Rees, P., Stillwell, J., Convey, A., Kupiszewski, M. (eds), Population Migration in the European Union, Chichester, Wiley.

POULAIN, M. 1999. Confrontation des statistiques de migration intra europennes: vers une matrice complete?, Eurostat Working Paper 5 Theme 3, Luxembourg, Eurostat.

SALT, J. 1983-4. "High Level Manpower Movements in Northwest Europe and the Role of Careers: an Explanatory Framework", International Migration Review, 17, 633-652

RECCHI, E., TAMBINI, D., BALDONI, E., WILliAMS, D., SURAK, K., FAVELL, A. 2003. Intra-EU Migration: $A$ Socio-demographic Overview, PIONEUR working paper 2003/2, Florence, CIUSPO.

RODRÍGUEZ POSE, A. 2002. The European Union: Economy, Society, and Polity, Oxford, Oxford University Press.

SALT, J. y SCHMIDT, S. 2001. "The Development of Free Movement in the European Union", en Papademetriou, D. and Meyers, D. W. (eds.), Caught in the Middle: Border Communities in an era of Globalisation, Washington, Carnegie Endowment Publications.

SANTACREU, O. y ALBERT, M.C. 2004. "Identidad Espacial y Simbólica en Europa" en Tezanos, J.F. (ed.) Tendencias en identidades, valores y creencias. Madrid, Editorial Sistema.

THOROGOOD, D. y WINQVIST, K. 2003. Women and Men Migrating to and from the European Union, Statistics in Focus Eurostat, 2, 1-8.

WARNES, A. y PATTERSON, G. 1998. "British Retirees in Malta: Components of the Cross national Relationship", International Journal of Population Geography, 4, 113-133. 